\title{
OPINION PATTERN MINING BASED ON PROBABILISTIC PRINCIPLE COMPONENT ANALYSIS REPORT
}

\author{
P.Saravana Kumar', A.Vijaya Kathiravan ${ }^{2}$ \\ ${ }^{1}$ Assistant Professor, Department of Computer Applications, K.S.Rangasamy College of Technology,Tiruchengode, \\ Anna University, Chennai. \\ ${ }^{2}$ Assistant Professor, Department of Computer Science, Govt.Arts College, Periyar University, Salem
}

\begin{abstract}
Now days, Customer feedback and satisfaction is playing a significant role in commercial product to market. Customer can be reviewed by other customer feedback and collect all the relevant information related to a particular product. Based on that the decision can be taken to purchase the product. In the traditional method, Random forest predicted the impact of the review but not worked with segmentation on the basis of multiple reviewer comments. At the same time, the variable cluster algorithm has been addressed in the market segmentation for retailing the customer's lifestyle. It has been provided with the segmentation method, but not guide to full proof strategies for different product decision. Instead of that to guide different customers with a variety of product feedback using pattern mining approaches. The product review pattern mining segmentation based on probabilistic principle component analysis is proposed. The opinion mining, segments has categorized into several segments with pattern analysis based on multiple review comments. This mechanism has reduced the dimensionality of the segmentation process using the covariance matrix approach. The experiment uses the opinion rank review dataset information for further process. It increases the segmentation efficient upto9\% when compare with traditional and conventional methods. The experimentation has been done with the important factor of opinion decision threshold, false positive rate, segmentation efficiency and customer product ratio level along with customer behavioral feedback.
\end{abstract}

Keywords: Covariance Matrix, Opinion Pattern Mining Segmentation, Probabilistic Principle Component Analysis, , Product Review

\section{INTRODUCTION}

Opinion mining is a process for tracking the mood of the public about a certain product. Segmentation has turned out to be the primary conceptual model both in marketing theory and in practice. With the increasing use of online reviews, customers post the reviews of the products and dedicated review sites. These reviews provide excellent sources for obtaining the opinions of the valuable consumers about the products, which are very useful to both potential customers and product manufacturers. Techniques are now being developed to exploit these sources to help companies and individuals to gain such information effectively and easily. Techniques are now being developed to exploit these sources to help companies and individuals to gain such information effectively and easily. Taiwan's economy as described in [10] accompanied a model in the country's developing market.

Discovery of customer relationship between huge databases has been recognized to be useful in discerning marketing, decision analysis, and business management. An important application area of opinion mining relationship is the market basket analysis, which demonstrates the buying behaviors of customers. The main idea behind the framework of pattern mining is to apply an efficient segmentation method that distinguishes the customer likeness and unlikeness of the product. By doing so, pattern mining helps to repeatedly determine the relative amount by obtaining the assessment results.

Spatiotemporal data representation in [5] followed the association rule for discovering the knowledge but the detailed customer requirements were not analyzed. The dimensionality class measure was though precise to each of the spatiotemporal data mining tasks but they were not identified with effective ratio. Spatio-Temporal Association Rules as presented in [7] developed an efficient algorithm to reduce the linear run time. The interesting and verifiable patterns were carried out using the world animal tracking data set but not suitable for business processing mode. Multiple-Instance Learning via Disambiguation (MILD) as described in [16] recognized the correct optimistic instances for business processing model.

Probabilistic Spatio temporal model for target event as demonstrated in [14] identified the midpoint of the product incident position but still advanced algorithms were not developed for query optimization. Twitter user is observed as a sensor and every tweet act as sensory information but the segmentation operation were not carried out. Multiple reviewer-level features as described in [1], helped to measures the reviewers comment with extent of mining subjectivity. Reviews have a fusion of objective with highly skewed sentence that were associated with the product sales. The product review tends to include the objective 
information. Random Forest predicted the impact of reviews but not worked with the segmentation for different user opinions.

In this work, focus is made on developing an effective Opinion Pattern Mining Segmentation (OPMS) process. The Opinion Pattern Mining performs the segmentation process based on the Probabilistic Principle Component Analysis. OPMS based on the PPCA report reduces the dimensionality to make the segmentation process very easier. The segmentation of user profile helps to easily segment the behavioral pattern with determination of the maximum likelihood. Probability Principle Component Analysis updates the product reviews with the increased threshold rate and reduced false positive rate.

\section{OPINION \\ PATTERN \\ MINING \\ SEGMENTATION \\ BASED \\ ON THE \\ PROBABILISTIC PRINCIPLE COMPONENT}

\section{ANALYSIS}

Opinion pattern mining with PPCA report aims to establish the review of different user behavioral with orientation results and produce the result with lesser false positive and increased threshold rate. The architecture diagram of opinion pattern mining segmentation based on PPCA is described in Figure 1.

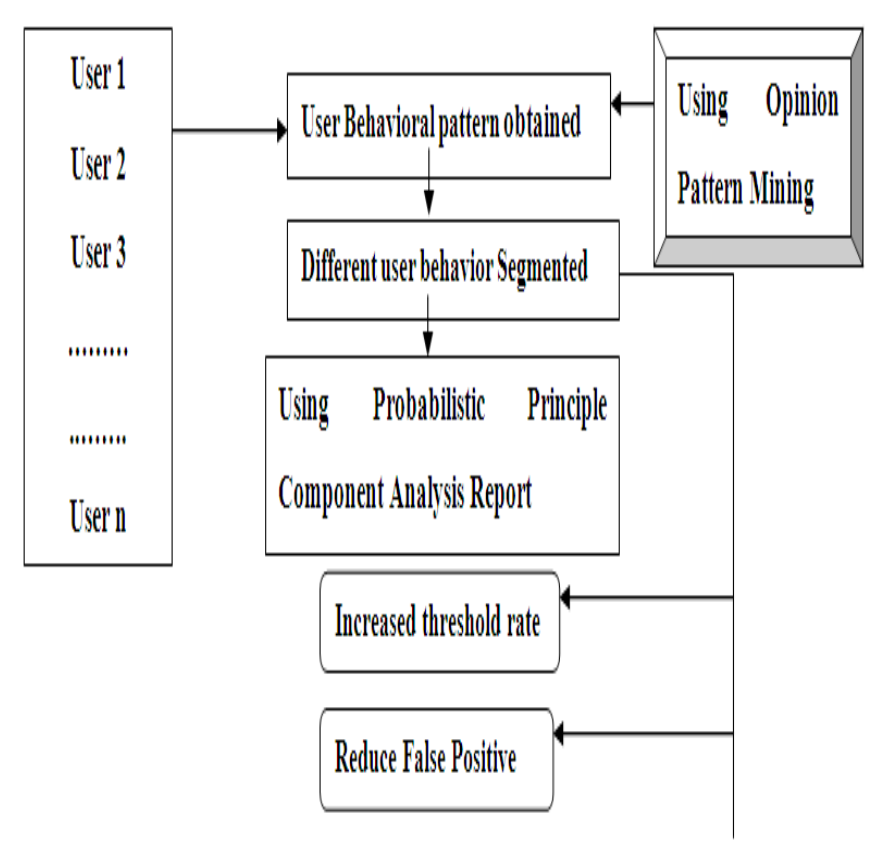

Fig 1 Architecture Diagram of PPCA on Opinion Pattern Mining

As illustrated in Fig 1, opinion pattern mining based on the probabilistic principle component analysis includes different user behavior. Different user behavior with the maximum likelihood is used for the estimation of orientation result. The user behavior is segmented using the Probabilistic Principle Component Analysis report. With the application of PPCA, the threshold rate is increased and decreases the false positive rate and also the dimensionality is reduced using the covariance matrix. The covariance matrix improves the segmentation efficiency and dimensionality reduction in PPCA further reduces the opinion pattern mining time.

\subsection{Probabilistic Principle Component Analysis}

\section{Report}

Based on the observation of the user behavior, PPCA report is obtained that exhibit lesser dimensionality while performing user behavior pattern segmentation. Each user behavior $\quad U 1, U 2, U 3 \ldots . U n$ represents different dimensionalities that have to be segmented for obtaining different patterns according to the user behavior. The center of dimensionality reduction in segmentation is formularized as,

$$
U_{n}=\frac{1}{n} \sum_{i=1}^{n} U_{i}-U_{i+1}
$$

$U_{n}$ denotes ' $\mathrm{n}$ ' user behavioral patterns whereas $U_{i}, U_{i+1}$ are the obtained behavior patterns of each user. Each user behavior is taken into account for providing efficient opinion pattern mining without any dimensionality reduction. Each user's carried out the step in (1) for efficiently segmenting the user behavioral by avoiding dimensionality reduction.

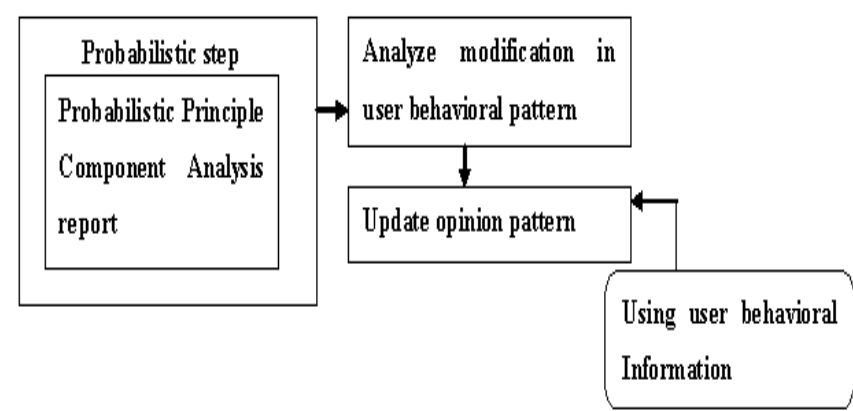

Fig 2: Probabilistic Procedure in PPCA

Fig 2 describes the step by step probabilistic usage in the PPCA. The probabilistic step in the PPCA report which is used to analyze and predict the modifications observed in the behavior of the user. The benefit of using the probability in PCA report is that it easily updates the likelihood value according to the changes observed in user behavior based on product reviewing.

PPCA covariance is the measure of variability co-exists that exists in user behavior pattern for opinion pattern mining. The ordered data move in the same pattern helps to reduce the dimensions. Each element represents the user (in a vector) identifies the behavioral pattern which is in the form of scalar arbitrary point. The scalar arbitrary point is in the form of finite number of observed empirical values specified by a theoretical joint probabilistic distribution for segmentation. 


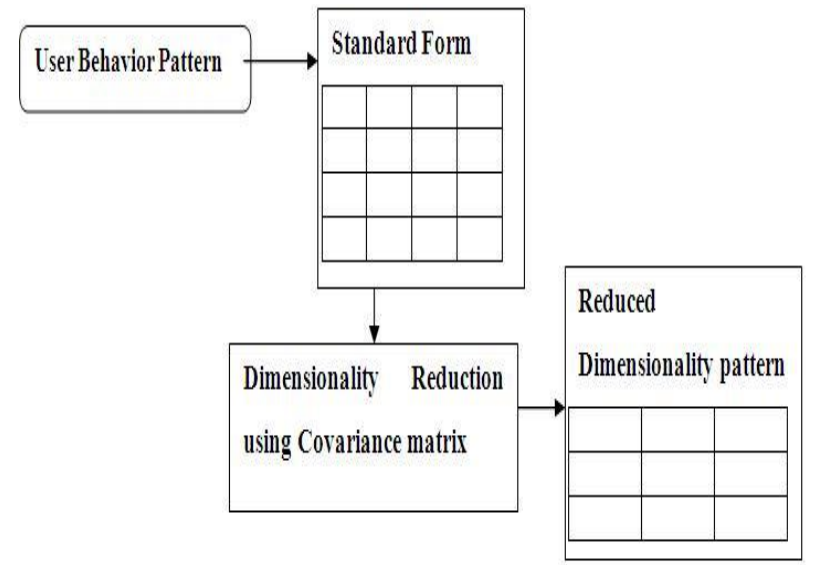

Fig 3 Dimensionality Reduction Procedure

Fig 3 reduces the dimensionality from the standard user behavioral pattern to the reduced dimensional pattern. Dimensionality represents the orthogonal projection and is attained using the covariance parameterization. PPCA report operates as an effective mechanism in opinion pattern mining segmentation with reduced dimensionality rate.

\subsection{Opinion Pattern Mining Segmentation}

Opining pattern mining segmentation with PPCA report performs the process of mining and segments the pattern efficiently without any redundancy. PPCA estimate the distribution of the pattern and segments effectively based on similar user behavior patterns. The algorithmic description of OPMS using the PPCA report is described as,

\section{//Opinion Pattern mining with PPCA report \\ Begin \\ Input: User Input pattern 'U1, U2, U3...Un'}

Output: Opinion Pattern mining with lesser false positive ratio

For Each User

Step 1: Analyze each user behavior from 'U1, U2, U3...Un'

Step 2: User behavior Segmented into 'S1', 'S2','S3, ...'Sn'

Step 3: Opinion pattern used to attain user product reviews follows

Step 3.1: Based on the maximum Likelihood $\frac{1}{n} \sum_{1}^{n+1} \lambda_{1}, \lambda_{2}, \ldots \ldots \lambda_{n}$ using Eigen Values

Step 3.2: Reduced the dimensionality using Covariance matrix

Step 3.3: Covariance matrix $\sum_{i, j}=\operatorname{cov}\left(x_{i}, x_{j}\right)=E\left[\left(x_{i}-\right.\right.$ $\left.\lambda_{i}\right)\left(x_{j}-\lambda_{j}\right)$ computed

Step 4: Probabilistic update the opinion of different user on different products

End For

Sep 5: Goto step 1

Step 6: Run P C A Analysis until user 'Un' End

In OPMS theory, the complex type of user queries is also segmented readily with the PPCA report. The segmented form of opinion pattern mining is illustrated the Fig4.

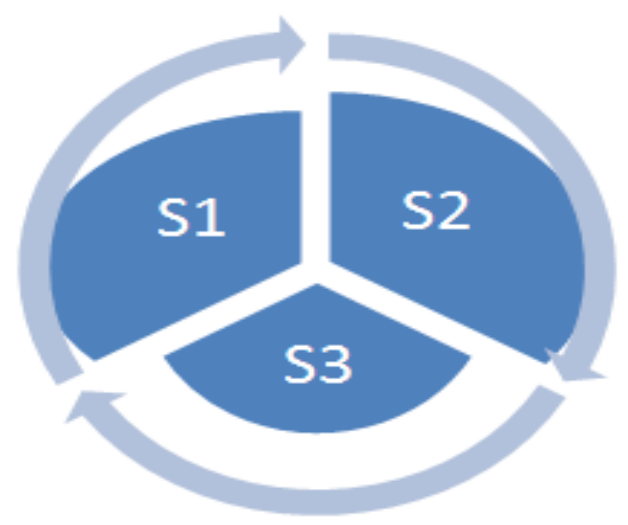

Fig 4 Segmented opinion pattern mining

The segmented part illustrates that similar opinions are grouped together using PPCA report. S1, S2, and S3 are the three set of segmented principles associated with the each user behavior pattern. Efficient segmenting of user profiles obtains the users behavioral patterns (i.e.,) opinion pattern mining with increased threshold rate and decrease the false positives.

Threshold Factor Analysis in OPMS $(T)=\sigma^{2} * i$

False Postive Facor Analysis $(F A)=1-(1-e)^{-s^{N}}$

The factor analysis is carried out using the segmented principles in the opinion pattern mining. The factor analysis with increased threshold rate improves the level of the user's product trend ratio and false positive rate reduced to improve the accuracy level on the user behavioral pattern mining.

\section{EXPERIMENTAL EVALUATION}

Opinion Pattern Mining Segmentation based on the Probabilistic Principle Component Analysis (PPCA) uses JAVA platform with Weka tool for the experimental work. PPCA report uses the OpinRank Review Dataset extracted from the UCI repository for the experimental work. The OpinRank dataset contains user reviews related to car and hotels. The information is collected from the Tripadvisor and Edmunds. The Tripadisor shows the 268000 reviews and Edmunds reviewed the 51,240 reviews.

OpinRank Review Dataset contains the full review of the car model from 2007. The review holds the 140-250 cars for each year. The review data extracted the fields, including the dates, author names, favorites and the full text review. The total review is expected to be 51,240. The review of the hotel for 10 different cities such as Dubai, Beijing, London, New York City, New Delhi, San Francisco, Shanghai, Montreal, Las Vegas, and Chicago are collected. OpinRank Review Dataset has about 80 to 700 hotels in each city. The total number of reviews on the hotel is expected to be 268,000 . 
The false positive ratio is the probability of incorrectly rejecting the null suggestion for particular dataset information. The false positive rate of the OPMS is defined as,

$$
\text { False positive Rate }=\left(\frac{V}{E_{0}}\right) * 100
$$

Where $\mathrm{V}$ denotes the false positive rate of the product and $E_{0}$ denotes the true result obtained from the customers. Segmentation is defined as the important concept in marketing to serve different types of customer. Segmentation efficiency is measured in terms of the success percentage (success \%).

$$
\text { Pattern Mining Time }=P 1-P 2
$$

Where $P 1$ represents the Start time of pattern construction and $P 2$ denotes the End Time of Pattern construction. Opinion pattern mining time is measured in terms of seconds $(\mathrm{Sec})$. Dimensionality reduction is measured as,

$$
D R=\frac{(\text { No. of dimensions }- \text { Reduced Dimensions })}{* 100}
$$

User product trend ratio level denotes the amount of accurate product review attained by using the opinion pattern mining, segmentation with the probability principle component analysis report.

\section{RESULT ANALYSIS}

Opinion Pattern Mining Segmentation based on the Probabilistic Principle Component Analysis (PPCA) is compared against the Random Forest based classifier (RF) method and Variable Clustering (VC) algorithm. OPMS is evaluated using the OpinRank Review Dataset from the UCI repository.

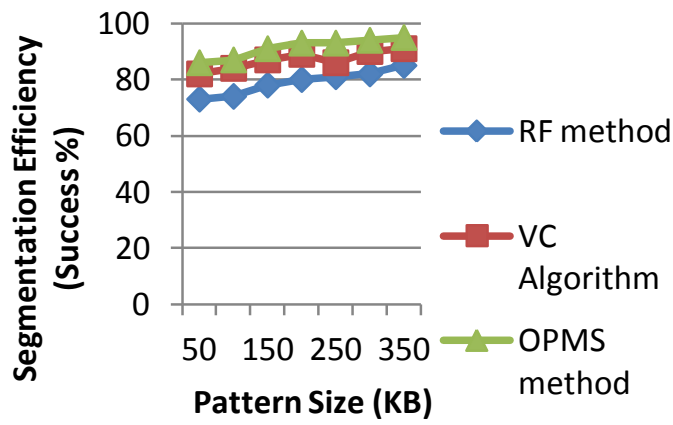

Fig 5 Segmentation Efficiency Measure

Fig 5 PPCA segments the user behavioral sequences automatically into diverse behaviors. The covariance matrix form improves the segmentation efficiency by $11-17 \%$ when compared with the RF method [1] and improved by 3 $-8 \%$ when compared with the VCAlgorithm[2].

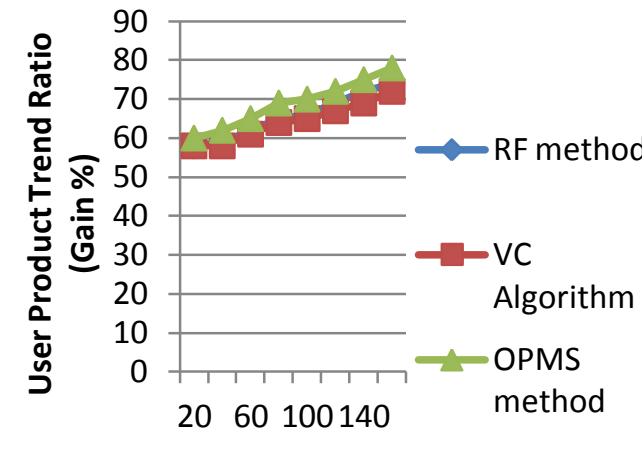

Fig. 6 User Product Trend Ratio Level Measure

Fig 6 The product reviewing with marginal distribution in PPCA report updates the opinion patterns. The marginal distribution is applied to update on the entire user behavioral pattern and attain $3-6 \%$ improved ratio level when compared with the RF method [1].

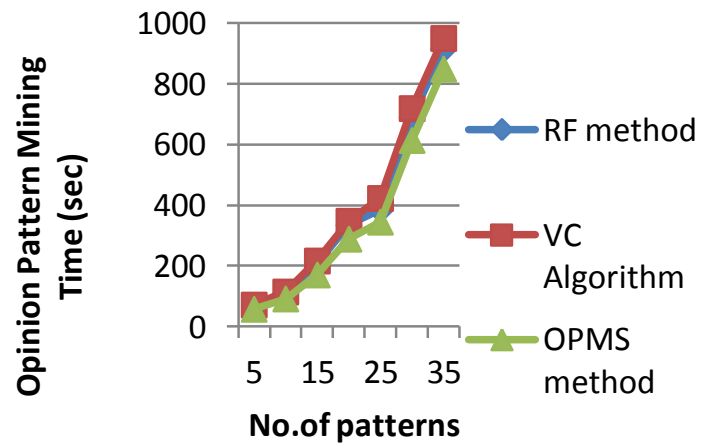

Fig 7 Opinion Pattern Mining Time Measure

Fig 7 In order to compute the mining process the value of ' $C M r$ ' is obtained. The reduction of dimensionality in OPMS method reduces the opinion pattern mining time by 7 - $17 \%$ when compared with the RF method [1]. The less correlated value reduces the pattern mining time by $10-21$ $\%$ in opinion pattern mining segmentation when compared with the VC Algorithm [2].

\section{CONCLUSION}

The proposed opinion pattern mining, segmentation approach based on the Probabilistic Principle Component Analysis is a precious method in which segments, the useful customer review information from large amounts of repository data in an efficient manner. Opinion pattern mining based segmentations show significance in data mining technologies to reduce the false positive rate in reasonably because the data organized with the maximum likelihood mapping of the users' behavior. The experiment result has produced that the opinion pattern mining,in which the segmentation outperforms all the existing segmentations work with $16.69 \%$ and improved decision threshold rate 
and system efficiency rate is also increased in the conventional method. The probability PCA updated the product reviews ratio level by $7.50 \%$ based on the users behavioral reviews. An Opinrank review dataset from the UCI repository is used to review the experimental result of OPMS with traditional method of the parametric factors such as opinion pattern mining time, false positive rate, and dimensionality reduction rate. The proposed mechanism has deployed it in the real time application fields such as online commercial products based on the customer review comments recommended. The proposed mechanism guaranteed to produce efficient results.

\section{REFERENCES}

[1]. Anindya Ghose., Panagiotis G. Ipeirotis., "Estimating the Helpfulness and Economic Impact of Product Reviews: Mining Text and Reviewer Characteristics," IEEE TRANSACTIONS ON KNOWLEDGE AND DATA ENGINEERING, 2010

[2]. V.L. Migueis., A.S. Camanho., Joao Falcao e Cunha., "Customer data mining for lifestyle segmentation," Expert Systems with Applications., Elsevier Journal., 2012

[3]. Archana Tomar., Vineet Richhariya., Mahendra Ku. Mishra., " A Improved Privacy Preserving Algorithm using the Association rule mining in centralized database.," International Journal of Advanced Technology \& Engineering Research (IJATER) ISSN NO: 2250-3536 VOLUME 2, ISSUE 2, 2012

[4]. Marco Muselli., and Enrico Ferrari.,"Coupling Logical Analysis of Data and Shadow Clustering for Partially Defined Positive Boolean Function Reconstruction," IEEE TRANSACTIONS ON KNOWLEDGE AND DATA ENGINEERING, VOL. 23, NO. 1, JANUARY 2011

[5]. K.Venkateswara Rao., A.Govardhan., and K.V.Chalapati Rao.," Spatiotemporal Data Mining:issues, tasks and Applications.," International Journal of Computer Science \& Engineering Survey (IJCSES) Vol.3, No.1, 2012

[6]. Swarupa Panmetsa., L.V.S.S., Ch. Raja Ramesh, "Anonymization of the Sequential Patterns in Location Based Service Environments," International Journal of Computer Technology \& Research, IJCTR, ISSN 23198184,Vol 1, Issue 1, October 2012

[7]. Florian Verhein., "Mining Complex Spatio-Temporal Sequence Patterns," Journal of Science, 2009

[8]. Ning Zhong., Yuefeng Li., Sheng-Tang Wu., "Effective Pattern Discovery for Text Mining," IEEE Transactions on Knowledge and Data Engineering, Volume:24, Issue: 1, Jan 2012

[9]. Eric Hsueh-Chan Lu, Vincent S. Tseng., and Philip S. Yu., "Mining Cluster-Based Temporal Mobile Sequential Patterns in Location-Based Service Environments,"IEEE TRANSACTIONS ON KNOWLEDGE AND DATA ENGINEERING, VOL. 23, NO. 6, JUNE 2011

[10]. Chih-Hao Wena.,, Shu-Hsien Liao., Wei-Ling Chang., Ping-Yu Hsu., "Mining shopping behavior in the Taiwan luxury products market," Expert Systems with Applications., Elsevier Journal., 2012

[11]. Emilio Miguelanez., Pedro Patron., Keith E. Brown., Yvan R. Petillot., and David M. Lane., "Semantic Knowledge-Based Framework to Improve the Situation
Awareness of Autonomous Underwater Vehicles," IEEE TRANSACTIONS ON KNOWLEDGE AND DATA ENGINEERING, VOL. 23, NO. 5, MAY 2011

[12]. Panagiotis Papadimitriou., Panayiotis Tsaparas, Ariel Fuxman., and Lise Getoor., "TACI: Taxonomy-Aware Catalog Integration," IEEE TRANSACTIONS ON KNOWLEDGE AND DATA ENGINEERING, VOL. 25, NO. 7, JULY 2013

[13]. Tao Jiang., and Ah-Hwee Tan., "Learning Image-Text Associations," IEEE TRANSACTION ON KNOWLEDGE AND DATA ENGINEERING., Volume:21 , Issue: 2, Feb 2009

[14]. Takeshi Sakaki, Makoto Okazaki, and Yutaka Matsuo.,"Tweet Analysis for Real-Time Event Detection and Earthquake Reporting System Development," IEEE TRANSACTIONS ON KNOWLEDGE AND DATA ENGINEERING, VOL. 25, NO. 4, APRIL 2013

[15]. Jae-Gil Lee., Jiawei Han., Xiaolei Li, and Hong Cheng., "Mining Discriminative Patterns for Classifying Trajectories on Road Networks," IEEE TRANSACTIONS ON KNOWLEDGE AND DATA ENGINEERING, VOL. 23, NO. 5, MAY 2011

[16]. Wu-Jun Li., and Dit-Yan Yeung., "MILD: MultipleInstance Learning via Disambiguation," IEEE TRANSACTIONS ON KNOWLEDGE AND DATA ENGINEERING, VOL. 22, NO. 1, JANUARY 2010

[17]. Oana Frunza., Diana Inkpen., and Thomas Tran., "A Machine Learning Approach for Identifying DiseaseTreatment Relations in Short Texts," IEEE TRANSACTIONS ON KNOWLEDGE AND DATA ENGINEERING, VOL. 23, NO. 6, JUNE 2011

[18]. Wenjing Zhang., and Xin Feng., "Event Characterization and Prediction Based on Temporal Patterns in Dynamic Data System," IEEE TRANSACTIONS ON KNOWLEDGE AND DATA ENGINEERING., 2013

[19]. Jung-Yi Jiang., Ren-Jia Liou., and Shie-Jue Lee., "A Fuzzy Self-Constructing Feature Clustering Algorithm for Text Classification," IEEE TRANSACTIONS ON KNOWLEDGE AND DATA ENGINEERING, VOL. 23, NO. 3, MARCH 2011

[20]. Liang Wang., Christopher Leckie., Kotagiri Ramamohanarao., and James Bezdek., "Automatically Determining the Number of Clusters in Unlabeled Data Sets," IEEE TRANSACTIONS ON KNOWLEDGE AND DATA ENGINEERING, VOL. 21, NO. 3, MARCH 2009

\section{BIOGRAPHIES}

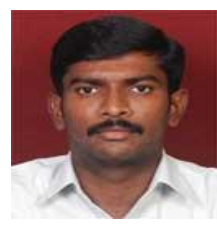

P.SARAVANA KUMAR, is currently working as an Assistant Professor in Master of Computer Applications in K.S.Rangasamy College of Technology,Tiruchengode,Namakkal. He is handling PG classes. $\mathrm{He}$ is also pursuing Ph.D., in Computer Science under Periyar University, Salem-11. He presented a paper in International conferences and also he published in International Journal. His area of interest is DataMining. 


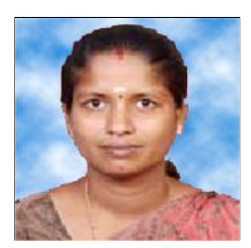

Dr.A.VIJAYA KATHIRAVAN is working as an Assistant Professor in Computer Applications in PG and Research Department of Computer Science, Govt. Arts College (Autonomous), Salem-07, TamilNadu, INDIA. She received her M.Phil. in Computer Science from Bharathiar University, Coimbatore and she awarded her doctoral degree in Computer Applications from University of Madras, Chennai. She has published 6 Books, 3 papers in National Journal, 30 papers in International Journal, 35 Papers in National Conference Proceedings, 38 Papers in International Conference Proceedings and a total of 112 publications. Her research interests include data structures and algorithms, data/text/web mining, search engines, web communities, social network mining, machine learning, Natural Language Processing, Organizational leadership and human resource management. 